\title{
Bežalec: The sport competition management software
}

\author{
Dušan Fister \\ Univerza v Mariboru \\ Fakulteta za strojništvo \\ Smetanova 17, Maribor \\ dusan.fister@ \\ student.um.si
}

\author{
Uroš Mlakar \\ Univerza v Mariboru \\ Fakulteta za elektrotehniko, računalništvo in \\ informatiko \\ Koroška 46, Maribor \\ uros.mlakar@um.si
}

\begin{abstract}
There is a need for automatic management software in every area. This paper proposes a simple software application for managing competitors registration, editing, exporting and generating their standings and printing certificates. QtCreator was used to handle the visualization and integration of different tasks. The managing software, called "Bežalec", has been practically tested on a local running competition and performed well enough.
\end{abstract}

\section{Keywords}

management software, automation, integration

\section{INTRODUCTION}

Automatic management software is intended to simplify the registration, generation of result lists and commemoration certificate printing for sport competitions. It is desired, that this software is completely automatic and minimum user intervention is required. The application has to offer some additional tasks, e.g. uploading result lists to the website. The software, in general, has to be suitable for running competitions, but should be extendible to cycling and swimming competitions and other sports with single criterion performed time.

This paper proposes the structure of an application, that was built for the purposes of a local running competition. This application was built in the $\mathrm{C}++$ programming language in programming environment QtCreator, under Linux operating system [2]. The Qt library was used for designing the graphical user interface (GUI) of the application. Besides the GUI design, awk, sed and Perl scripts were integrated into the main application, as well as MySQL database. Some other components are included into the applications, e.g. $l p$ printing tool, pdflatex and html generator. The application is currently suitable for running competitions, as shown in logo (Fig. 1).

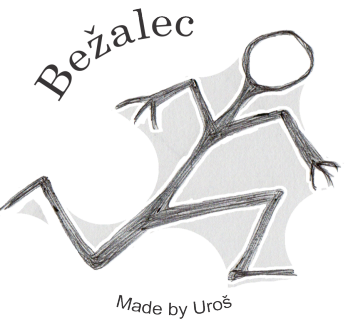

Figure 1: The management software "Bežalec" logo.

The structure of this paper is as follows: in the second chapter, the sport management software and its parts are described. In the third chapter, a short discussion is outlined regarding the usability of the application and the potential to extend it for using in other sports. The paper is concluded in chapter 4.

\section{THE SOFTWARE}

The software was written, as already mentioned, in QtCreator programming environment. The product of source code is a single executable script, which runs the following main dialog window, shown in Fig. 2. It is worth to mention, that the management software is completely operated from this dialog window. The dialog window consists of two pages, i.e. first "Registracija" page and second "Vnos" page. Names are written in Slovene and therefore require translations.

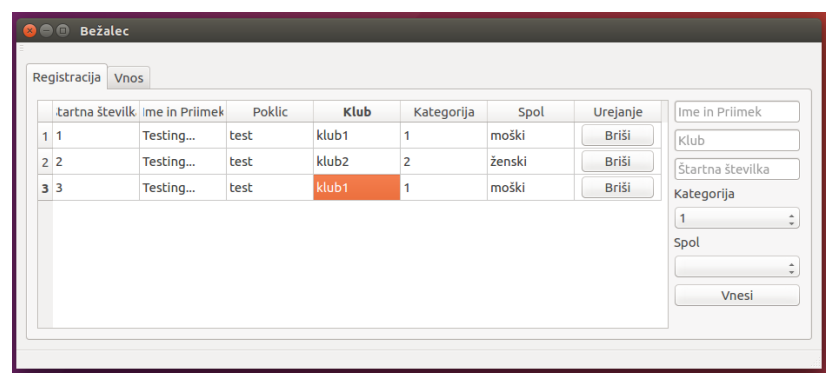

Figure 2: The management software main dialog window.

The first page of the shown main dialog window is dedicated for registration of competitors - athletes. They are organized in a list in tabular way, with each athlete occupying one row of the table. Basic data about every athlete is taken, as following: 
- starting number,

- name and surname,

- profession,

- club name,

- category and

- sex.

Those data are inputed into the management software via input fields on the right side of the main dialog window, shown in Fig. 2. After confirming input data, the athlete is added to the list, as well as exported into a text file and a MySQL database for back-up [1]. Details are given in subsection 2.2 .

Every registered athlete is then treated by an incremental starting number, which is her/his identification number (ID). Data about an athlete can be, after successful registration, easily changed for any modifications. Athletes can be, in any case, removed from the registration list.

The main display window is extended by an auxiliary display window, shown in subsection 2.1. The main display window also contains the toolbar, from which basic settings can be arranged and the auxiliary window can be invoked.

\subsection{Treating the categories}

If basic data about athletes are standardized for every competition, categories however are not. Different sport competitions may have different ranges for age categories, e.g. some competitions rank young athletes into an age category from fifteen to twenty-nine years (M15-29), while some into under twenty-three (U23). This problem was solved by a custom category list, by assigning each category with a unique incremental ID. Fig. 3 shows the auxiliary dialog window for assigning categories.

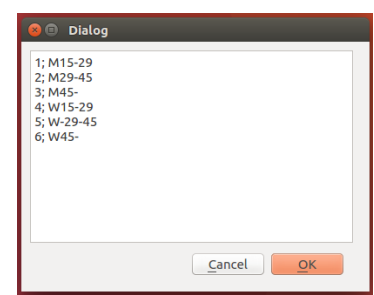

Figure 3: Auxiliary dialog window for assigning categories.

From the Fig. 3 it is seen, that any number of categories can be included, or added into the application, separated by a unique ID.

\subsection{Import/export of registration data}

The desire for importing/exporting athlete's data into a text file is applicable for easier manipulation of registration. Some competitions have an online pre-registration, where athletes register a few days before via Internet. It is therefore possible for a manager of this software to assemble pre-obtained data before the competition at home.
After his work, the latest text file is generated, which can be imported on other computers. Generated text file is for computers without an installed MySQL database an essential tool, since it stores the data over a longer period of time.

Exporting athlete's registration data consists of two variants:

- exporting data into a text file and

- exporting data into a MySQL database.

The manner of using the MySQL database, in our case, is to back-up data, written in the text file. It is well-known, that MySQL databases offer different automatic functions over data, e.g. sorting, which is highly desired for organizing the start list. In case of a main dialog window error, it can restore data and guarantee other basic manipulations with data. Firstly, it is however necessary for the manager to install the MySQL database. The auto-generated MySQL table offers an outlook, as illustrated in Fig. 4.

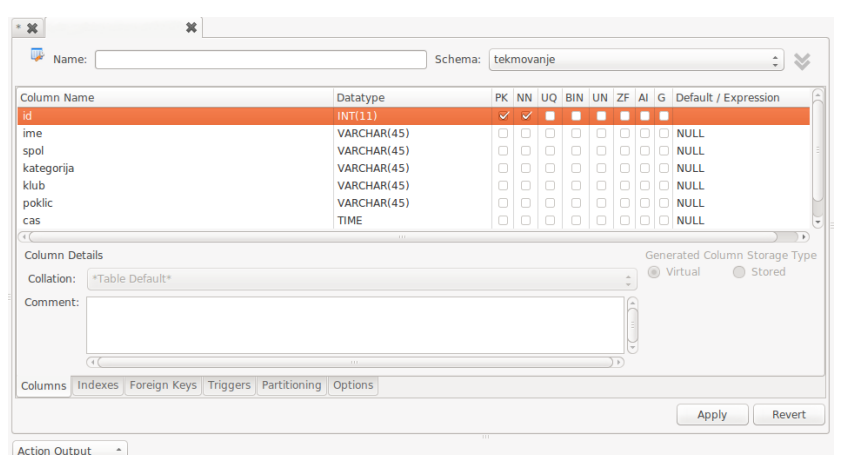

Figure 4: Snapshot from the MySQL database.

From the obtained data and sorting of starting numbers, a start list can be generated.

\subsection{Editing athlete's standings and result list generation}

After the competition, it is necessary to input athlete's results. They are stored in the form of time in following format: hh:mm:ss. This task is performed on the second page of the main dialog window, which is illustrated in the Fig. 5.

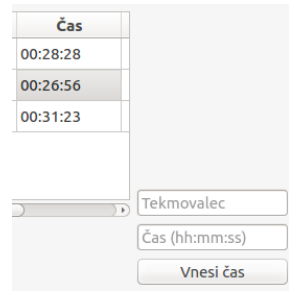

Figure 5: Second page for inputting results.

Two input fields are used for storing results, i.e. the unique ID of an athlete and his/her result. Using results from all athletes, a result list is generated, according to their performed time. Results are, besides shown in the table on 
the second page, also exported into the text file and the MySQL database. The following variants for generating the result lists are implemented:

- general classification results (for men and women) and

- age group results (for men and women).

General classification results consist of all athletes (who finished the competition), while age group results comprise only of athletes of suitable age. The difference between general classification results and age group results is shown in Fig. 6a and Fig. 6b.

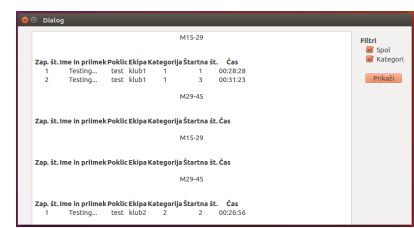

a)

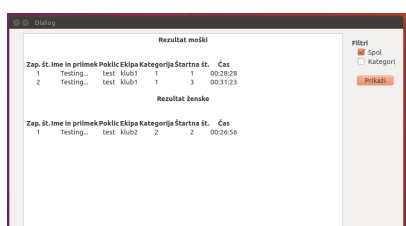

b)
Figure 6: Difference between the general classification and age group results.

\subsection{Printing commemorative certificates}

The local running competition provides commemorative certificates for their athletes. Thus, we would like to create them and print in a suitable format. Hence, we employed sed and awk tools for automatic editing of athlete's name, result and category in a pre-prepared latex source file, before a pdflatex tool was run under Linux operating system. The created certificate was printed using the $l p$ printing tool in the next step. Fig. 7 displays the created commemorative certificate. Due to keeping rights of the competition, the figure is retouched.

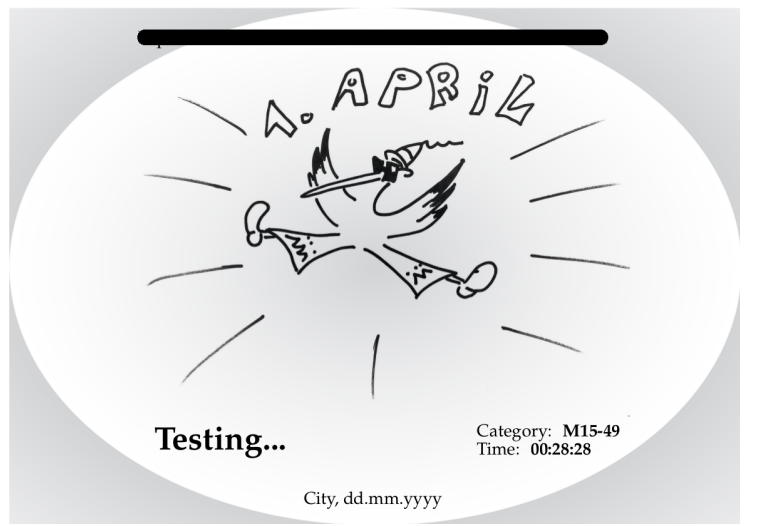

Figure 7: Automatically created commemorative certificate.

The whole "Bežalec" software package can be summarized by a use-case diagram in Figure 8 .

\subsection{Upload to the web page}

In the end of the athlete's result list manipulation, it is desired that results are automatically uploaded to the website.

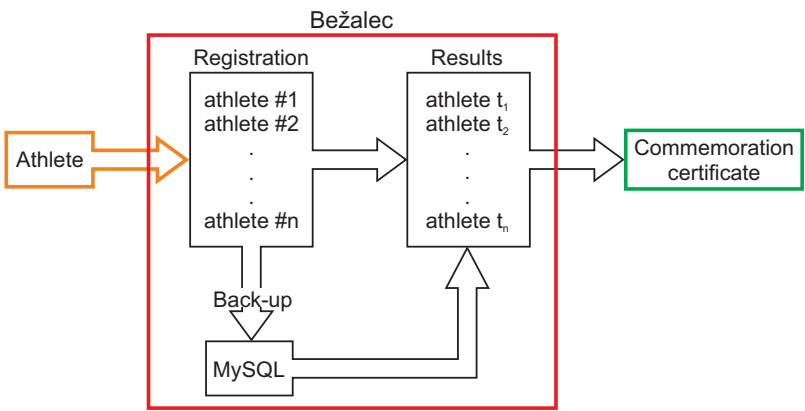

Figure 8: The use-case diagram for software package "Bežalec".

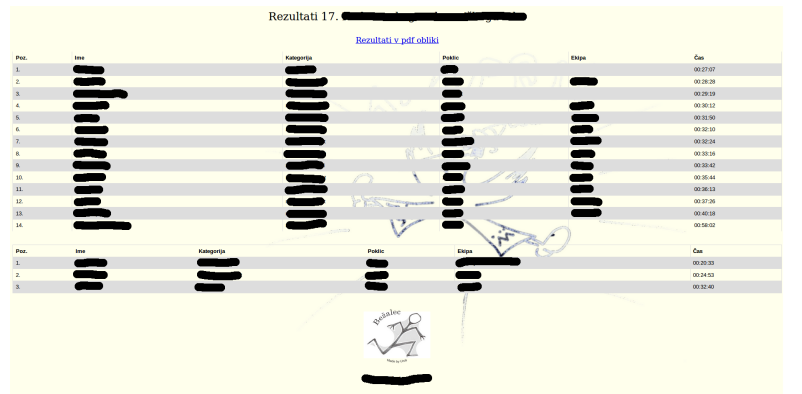

Figure 9: Automatically uploaded results list.

For that reason, a Node.js JavaScript run-time environment was used [3].

Fig. 9 (which is retouched, too) shows the uploaded result list in a html format (pdf format is opened in a new tab).

\section{DISCUSSION}

The idea of a sport management software does not merely present an application, but has a higher point of view. It can be upgraded by an autonomous chip-measuring equipment, where athletes run over a magnetised carpet and trigger an interrupt, which means precisely athlete's performed time (integration of chip-measuring equipment with management software was primarily our goal). The structure of a proposed automatic measuring system is shown in Fig. 10. It remains our goal for future work.

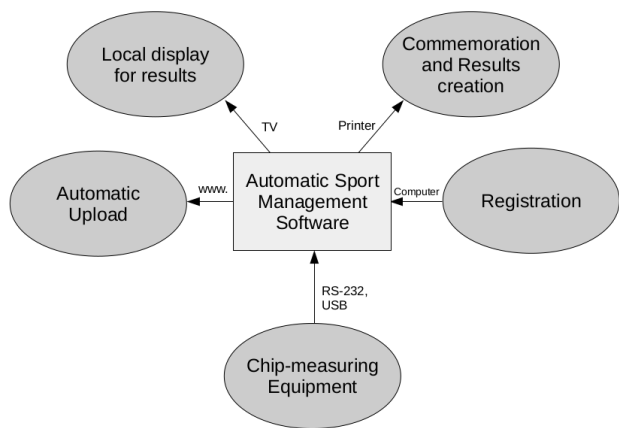

Figure 10: The structure of automatic measuring system. 


\section{CONCLUSION}

The application of sport management software has been tested on a local running competition, where seventeen athletes participated. The application successfully served during the registration, but stopped working after the start. However, the database provided a back-up and all data were restored. In this case, it was important, that MySQL database was employed for a back-up.

\section{REFERENCES}

[1] Oracle Corporation. MySQL 5.7 Reference Manual. Accessible at:

https://dev.mysql.com/doc/refman/5.7/en/. Accessed: 29.8.2017.

[2] R. Rischpater. Application development with qt creator. Packt Publishing Ltd, 2013.

[3] S. Tilkov and S. Vinoski. Node. js: Using javascript to build high-performance network programs. IEEE Internet Computing, 14(6):80-83, 2010. 